\title{
Microwetting of $\mathrm{pH}$ Sensitive Surface and Anisotropic $\mathrm{MoS}_{2}$
}

\section{Surfaces Revealed by Femtoliter Sessile Droplets}

Zhenzhen Lu, ${ }^{\dagger}$ Ziyang Lu, ${ }^{*}$ Shuhua Peng, ${ }^{+}$Xuehua Zhang ${ }^{*}{ }^{*}$ and Qingxia Liu ${ }^{*}{ }^{+}$

$\uparrow$ Department of Chemical and Materials Engineering, University of Alberta, Edmonton, AB, T6G 1H9, Canada. $\$$ Soft Matter \& Interfaces Group, School of Engineering, RMIT University, Melbourne, VIC 3001, Australia.

When oil forms a spherical-cap shape droplet on solid surface in water, the oil contact angle $\theta$ can be determined directly with the height $\mathrm{H}$ and lateral diameter L by Eq. $\mathrm{S} 1$.

$\sin \theta=\frac{\frac{1}{2} L}{R}=\frac{\frac{1}{2} L}{\frac{H^{2}+\frac{1}{4} L^{2}}{2 H}}=\frac{H / L}{(H / L)^{2}+\frac{1}{4}}$

When $\mathrm{H} / \mathrm{L}$ ratio is much smaller than 1 ,

$\sin \theta \cong 4 \times(H / L)$

It suggests that when $\mathrm{H} / \mathrm{L}$ ratio is much smaller than 0.5 , higher $\mathrm{H} / \mathrm{L}$ ratio will give larger contact angle.

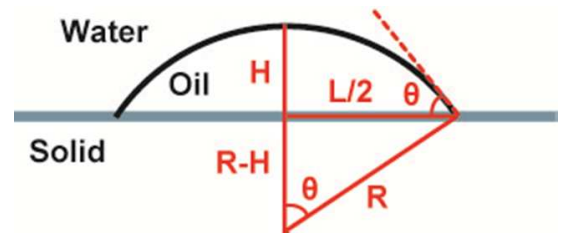

Fig. S1. A sketch of the geometry of oil droplet forming on solid surface in water. $H$ is height, $R$ is radius of curvature. 